\title{
Epigenetic Silencing
}

National Cancer Institute

\section{Source}

National Cancer Institute. Epigenetic Silencing. NCI Thesaurus. Code C48186.

This process is initiated by the formation of heterochromatin which ultimately leads to transcriptional inactivation or silencing. Once formed, heterochromatin can be inherited through many cell divisions, including meiosis. Inappropriate gain of heterochromatin structure, such as the inactivation of tumor suppressor genes by DNA hyper-methylation at their promoter regions, can be directly linked to carcinogenesis. 